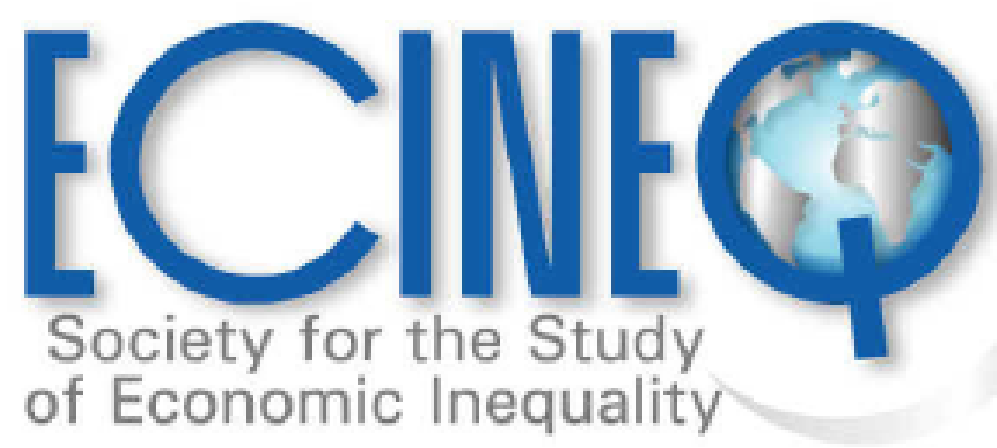

Working Paper Series

Distance-based social index numbers: a unifying approach

Walter Bossert

Conchita D'Ambrosio

Shlomo Weber 


\title{
ECINEQ 2020550
}

2020 July

www.ecineq.org

\section{Distance-based social index numbers: a unifying approach}

\author{
Walter Bossert \\ University of Montreal \\ Conchita D'Ambrosio \\ University of Luxembourg \\ Shlomo Weber \\ New Economic School, Moscow
}

\begin{abstract}
We present a unified approach to the design of social index numbers. Our starting point is a model that employs an exogenously given partition of the population into subgroups. A general parameterized class of group-dependent measures of deprivation is characterized, based on the differences between the income (or wealth) levels of an individual and those who are better off. We then continue to show that the general structure of our class is capable of accommodating a plethora of indices, including measures of inequality and polarization as well as distance-based measures of phenomena such as diversity and fractionalization.
\end{abstract}

Keyword: Group-dependent deprivation, inequality, polarization, diversity, fractionalization, social index numbers.

JEL Cassification: D31, D63. 


\title{
Distance-based social index numbers: a unifying approach*
}

\author{
WALter Bossert \\ CIREQ, University of Montreal \\ walter.bossert@videotron.ca \\ Conchita D'Ambrosio \\ University of Luxembourg \\ conchita.dambrosio@uni.lu \\ Shlomo WeBer \\ New Economic School, Moscow \\ sweber@nes.ru
}

This version: September 18, 2020

\begin{abstract}
We present a unified approach to the design of social index numbers. Our starting point is a model that employs an exogenously given partition of the population into subgroups. A general parameterized class of group-dependent measures of deprivation is characterized, based on the differences between the income (or wealth) levels of an individual and those who are better off. We then proceed to show that the structure of our class is sufficiently general to accommodate a plethora of indices, including measures of inequality and polarization as well as distance-based measures of phenomena such as diversity and fractionalization. Journal of Economic Literature Classification Nos.: D31, D63.
\end{abstract}

Keywords. Group-dependent deprivation, inequality, polarization, diversity, fractionalization, social index numbers.

* Financial support from the Fonds de Recherche sur la Société et la Culture of Québec and the Fonds National de la Recherche Luxembourg (Grant C18/SC/12677653) is gratefully acknowledged. 


\section{Introduction}

A wide range of social index numbers are based on comparisons among individuals along various socially relevant characteristics such as income and wealth, ethnicity, language and religion. One reason for this phenomenon is the realization that individuals do not live in isolation and compare themselves with others to understand their standing in society or to evaluate the degree of societal diversity; see Clark and D'Ambrosio (2015) for a comprehensive survey. These indices have appeared in many contributions and relate to different characteristics of societies, including deprivation, inequality, polarization, fractionalization and diversity. Our objective is to show that these substantially different concepts can be unified within the same basic structure.

The notion of a sense of deprivation that may arise from these comparisons appears first in Runciman (1966, p. 10) who writes "[w]e can roughly say that [a person] is relatively deprived of X when (i) he does not have X; (ii) he sees some other person or persons, which may include himself at some previous or expected time, as having X, (iii) he wants X, and (iv) he sees it as feasible that he should have X." On the same page, Runciman (1966) adds that "[t]he magnitude of relative deprivation is the extent of the difference between the desired situation and that of the person desiring it." Deprivation has been introduced in the Economics literature by Yitzhaki (1979) who proposed an index that continues to be one of the most (if not the most) widely used measure in the requisite literature. Hey and Lambert (1980) phrase the Yitzhaki index in terms of the income differences between an individual and those who are better off; see also Yitzhaki (1980). The importance of designing a suitable measure of deprivation is underlined by its role as one of the main driving forces behind major social phenomena, including crime (Kawachi, Kennedy and Wilkinson, 1999), political violence (Gurr, 1968) and migration decisions (Stark and Taylor, 1989).

Indices of individual deprivation naturally constitute a platform for aggregate societal indices. The first, and still the most remarkable, contribution in this vein is to be credited to Gini (1912). In his book Variability and Mutability, published in Italian, Gini (1912) provides a thorough examination of societal diversity and introduces two main groups of measures, one for each of the types of phenomena that his work focuses on. The first part of his book is devoted to indices of variability where he analyzes distributions of economic variables such as income or wealth. In the second part of his monograph, he moves on to what he refers to as indices of mutability with a focus on social variables that address notions of fractionalization, linguistics or ethnic diversity, among others.

The two types of indices introduced by Gini continue to have a profound impact on theoretical and empirical research to examine the consequences of diversity on various economic and societal outcomes; a thorough review is provided by Ginsburgh and Weber (2020). The first of his measures consists of the well-known relative Gini coefficient designed to measure the degree of inequality in an income or wealth distribution and its absolute counterpart. Yitzhaki (1979) provides the interesting and thoughtful observation that the value of the absolute Gini coefficient is equal to his aggregate measure of deprivation. Gini's second index is what is now called the Gini-Simpson fractionalization index; see, in addition to Gini (1912), Simpson (1949). This measure extends the notion 
of deprivation beyond income differences to include attributes such as ethnicity, language or religion. It is worth noting that the Gini-Simpson index can be expressed as one minus the Herfindahl-Hirschman index of industrial concentration; see Hirschman (1945) and Herfindahl (1950).

While both of Gini's indices rely on the concept of differences (or, more generally speaking, distances), their conceptual underpinnings are quite different. In the case of incomes, it is universally agreed that, from the viewpoint of an individual, a higher income is better than a lower income. This is not necessarily the case in the framework of fractionalization, an area in which such a simple uni-dimensionality is absent - and in which there is no agreement that there is a well-defined notion of betterness; in fact, it is impossible to even begin to think of better and worse groups in this context. It is therefore not too surprising that the theoretical and empirical examination of such diverging indices has largely proceeded through separate channels. It is only in some recent contributions that there appear first attempts to link the Gini coefficient to ethno-linguistic diversity; examples of such approaches are Esteban and Ray (2011) and Alesina, Michalopoulos and Papaioannou (2016).

Esteban and Ray (1994) develop a theory for the measurement of income polarization based on the absolute Gini coefficient. In a multi-group setting, they introduce an alienation-identification framework according to which the members of different groups are alienated from each other while, within each group, a sentiment of identification prevails. Esteban and Ray (1994) modify the absolute Gini index by introducing an interaction term that is intended to capture the notion of clustering around the mean values of the various income groups. Duclos, Esteban and Ray (2004) extend the applicability of this measure and illustrate how the original measures of Esteban and Ray (1994) can be generalized to notions of distance that may be very different from income gaps. Prominent examples include distances between ethnic groups or the phenomenon of social polarization that may be mediated by income differences.

The primary objective of our contribution is to provide a unified approach that encompasses the measurement of social phenomena such as income inequality, deprivation, polarization, fractionalization and diversity. Our basic model assumes that there is an exogenously given partition of the population into subgroups; the criterion used to define group membership is left open so that the approach is universally applicable to any arbitrary group structure. In our baseline result, we assume that the variable the distribution of which is to be assessed is individual income - or, indeed, any one-dimensional attribute that can be unambiguously ranked (in the sense that more is better). We already pointed out the important role played by the sentiment of individual deprivation in the development of the theory of social index numbers so it is only natural that a class of deprivation measures forms the cornerstone of our analysis. Because an individual experiences a sense of deprivation when comparing his or her position with those who are better off, an examination of the phenomenon seems suitable in the context of an unambiguously ranked variable. What is more surprising is that the general class of deprivation measures that we propose and characterize encompasses, in addition, conceptually very different phenomena such as fractionalization and diversity, where a unique measuring rod cannot but be absent. 
We begin with a characterization of a class of individual measures of deprivation in Section 2. Our indices generalize Yitzhaki's (1979) well-established measure by allowing for the existence of a group structure and, naturally, the Yitzhaki index itself emerges as a special case if there is but a single group. As is typically the case in this literature, the deprivation experienced by an individual is determined by the differences in incomes (or wealth) between the person under consideration and everyone in society who is richer (in the sense of having a higher level of income or wealth). These differences are weighted where the parameters (the weights) may differ across groups - that is, the income difference between the person in question and another member of society may depend on the groups these two individuals belong to. We do not restrict the relationship between different parameter values in any way; this is in line with the observation that there is no natural 'ranking' of the groups - all we know is that they differ and they may share different degrees of similarity. In this sense, our measures are very general and allow for a large variety of index numbers as special cases. Almost all of the properties that we employ in our characterization are well-established and accepted in the literature. The only axiom that is new refers to the group structure that is novel to our approach, and it is very mild and intuitive indeed - it merely requires that the index treats all individuals within each group symmetrically, paying no attention to their identities. Thus, the class of measures that we axiomatize rests on a solid theoretical and conceptual foundation.

In the remainder of Section 2, we move from individual deprivation measures to aggregate indices. As is common in the literature, we arrive at a societal measure of deprivation by calculating a weighted mean of the individual deprivation levels. This is a widely accepted method because the sentiment of deprivation is one that is experienced by individuals and, therefore, averaging is a perfectly legitimate operation. We stress that the weights we employ respect the essential requirement that members of the same group be treated symmetrically.

Section 3 is devoted to a discussion of special cases of our class. The rich variety of examples that we exhibit demonstrate that the measures we propose are remarkably accommodating indeed. The common theme that runs through all applications is that the requisite measures are based on the notion of distance - differences in the case of monetary variables, potentially more elaborate notions of distance (between groups, for instance) in more complex environments without a common unambiguous ranking. This suggests that our measures are absolute (in the sense of satisfying the well-know translation-invariance property), which is indeed the case. However, as is the case for much of this literature, relative notions can easily be accommodated - taking logarithms is a commonly-applied method to do so.

The section begins with the most naturally emerging special cases. In particular, we show that the indices of income polarization introduced by Esteban and Ray (1994) are obtained by a suitable choice of the parameter values. Although Esteban and Ray do not include it among their indices because they focus on the measurement of polarization rather than inequality, the absolute Gini (1912) coefficient is a member of our class as well. It is not too surprising that these measures appear as special cases because they are, as are measures of deprivation, based on income differences.

We then move on to more complex environments in which a single unambiguously 
ranked variable is not present. The fundamental difference is that these measures are based on general distance functions as compared to the natural distance that emerges as the difference of income or wealth levels in the earlier examples. We show that a large class of fractionalization indices can be expressed by means of the structure developed for our deprivation measures, although it is clear that they are not special cases in the strict sense of the term because they do not operate on income distributions; conceptually, however, they follow the pattern described by the members of our class. This class contains itself numerous special cases of interest, as we show subsequently in that section. In particular, the Gini-Simpson index (also referred to as Greenberg's, 1956, A-index in the literature) and the measure introduced by Davydov and Weber (2016) which generalizes the index of Reynal-Querol (2002) are included. In addition, we discuss Greenberg's (1956) B-index and some of its generalizations and variations developed in contributions by Davydov and Weber (2016) and Desmet, Ortuño-Ortín and Weber (2017). We then proceed with indices that combine income distributions with information on ethnic, linguistic or religious groups, leading to the diversity-inequality indices of Desmet, Le Breton, Ortuño-Ortín and Weber (2011), Chakravarty (2015) and Hodler, Srisuma, Vesperoni and Zurlinden (2020).

The section is concluded with an extension of the polarization measures of Esteban and Ray (1994) that combines polarization and a notion of diversity. This index has, to the best of our knowledge, not appeared in the previous literature. Thus, in addition to providing a unifying framework for a plethora of social index number, our contribution illustrates how our class can be employed to generate previously unidentified measures that may turn out to be very useful, especially in view of the current movement towards the integration of various social phenomena. We stress, again, that all of these measures can be accommodated within the conceptual framework provided by our fundamental class of deprivation indices.

We conclude the paper with Section 4 where we address some generalizations of our approach and mention a few measures that cannot be accommodated in our setting. In particular, our class does not include indices of ordinal inequality (Allison and Foster, 2004) and polarization (Apouey, 2007, and Permanyer and D'Ambrosio, 2015) since there is no natural ranking of the groups in our setting. In addition, indices that appear in the literature on measuring communication benefits in a multi-linguistic setting (see, for instance, the contributions by Selten and Pool, 1991, Church and King, 1993, Lazear, 1999, and Gabszewicz, Ginsburgh and Weber, 2011) fail to be members of our class because the group structure that they are based on does not provide a partition of the population.

\section{Group-dependent deprivation}

We begin our analysis with a characterization of a general class of individual measures of deprivation that forms the centerpiece of our contribution. To do so, we need some notational conventions. The set $N=\{1, \ldots, n\}$ with $n \geq 2$ is the set of individuals. The population is assumed to be partitioned into groups on the basis of some exogenously given 
criterion. Suppose that there are $K \in\{1, \ldots, n\}$ groups and let $S=\left\{S^{1}, \ldots, S^{K}\right\}$ be the requisite partition of $N$. We define $n^{k}=\left|S^{k}\right|$ for all $k \in\{1, \ldots, K\}$ so that $n=\sum_{k=1}^{K} n^{k}$. The group to which individual $j \in N$ belongs is $S^{k_{j}}$. For concreteness, we think of the variable in question as individual income but, clearly, our framework is applicable to any one-dimensional variable with a universally agreed-upon directional interpretation-more of it is better than less. For $x \in \mathbb{R}_{+}^{n}$ and $k \in\{1, \ldots, K\}, x^{k} \in \mathbb{R}_{+}^{n^{k}}$ is the subvector of $x$ the components of which are identified by the members of $S^{k}$. We also use the notation $x=\left(x^{k}\right)_{k \in\{1, \ldots, K\}}$. For $k \in\{1, \ldots, K\}, x^{k} \in \mathbb{R}_{+}^{n^{k}}$ and a permutation $\pi$ of $S^{k}, x_{\pi}^{k}$ is the distribution induced by applying the permutation $\pi$ to the components of $x^{k}$. The origin of $\mathbb{R}^{n}$ is denoted by $\mathbf{0}^{n}$, and $\mathbf{1}^{n}$ is the vector consisting of $n$ ones. For $x, y \in \mathbb{R}_{+}^{n}$ and a subset $M$ of $N$, the vector $x=\left(\left.y\right|_{M},\left.z\right|_{N \backslash M}\right)$ is defined as follows. For all $i \in N$,

$$
x_{i}= \begin{cases}y_{i} & \text { if } i \in M \\ z_{i} & \text { if } i \in N \backslash M .\end{cases}
$$

A group-dependent deprivation index for individual $i \in N$ is a function $D_{i}: \mathbb{R}_{+}^{n} \rightarrow \mathbb{R}_{+}$. As is standard in the literature on deprivation measurement, what matters from the viewpoint of individual $i$ are those whose incomes are higher than his or her own. Thus, for any distribution $x \in \mathbb{R}_{+}^{n}$, we define the set of those who are better off than $i$ as $B_{i}(x)=\left\{j \in N \mid x_{j}>x_{i}\right\}$. Because of our focus on groups, we require an analogous definition that keeps track of the groups the richer individuals belong to. Therefore, for $k \in\{1, \ldots, K\}$ and $x^{k} \in \mathbb{R}_{+}^{n^{k}}$, we define the set $B_{i}^{k}\left(x^{k}, x_{i}\right)=\left\{j \in S^{k} \mid x_{j}>x_{i}\right\}$. The latter set consists of the members of group $S^{k}$ whose incomes exceed that of individual $i$. In one of our examples, we also use the set $W_{i}(x)=\left\{j \in N \mid x_{j}<x_{i}\right\}$ of those who are worse off than $i$ in the distribution $x \in \mathbb{R}_{+}^{n}$.

We characterize the following class of measures. Let $w_{i}=\left(w_{i}^{1}, \ldots, w_{i}^{K}\right) \in \mathbb{R}_{++}^{K}$ be a vector of weights assigned to the constituent sets of our partition. The measure $D_{i}^{w_{i}}$ is defined by letting, for all $x \in \mathbb{R}_{+}^{n}$,

$$
D_{i}^{w_{i}}(x)=\sum_{k=1}^{K} \sum_{j \in B_{i}^{k}\left(x^{k}, x_{i}\right)} w_{i}^{k}\left(x_{j}-x_{i}\right)
$$

where we use the notational convention that a sum over an empty set is equal to zero. According to this measure, the sentiment of deprivation of individual $i$ is generated by a comparison with those who are richer. The level of deprivation experienced is mediated by the weights individual $i$ assigns to the groups that form the partition of the population.

Of course, by a suitable choice of the $w_{i}^{k}$, this class can alternatively be parameterized by expressing it as an arithmetic mean. Because we assume the overall population size $n$ and the group sizes $n^{k}$ to be fixed, replacing each $w_{i}^{k}$ with a ratio $c_{i}^{k} / n$ leads to an equivalent formulation. The same remark applies to ratios that involve dividing by $n^{k}$.

Our class represents a natural extension of Yitzhaki's (1979) index of individual deprivation to an environment in which the population is partitioned into exogenously given groups. The individual Yitzhaki index is obtained for $K=1$ and $w_{i}^{1}=1 / n$. 
The main result of this section is a characterization of these measures. We employ the following axioms, most of which are familiar from the traditional literature on deprivation without an external group structure.

Our first condition is a focus axiom, requiring that the income levels of those who are at or below that of person $i$ are irrelevant. This property is analogous to the focus axiom employed by Sen (1976) in his analysis of the measurement of poverty. It formalizes the requirement that the comparison group for individual $i$ consists of all those who are richer than him or her.

Focus. For all $x, y \in \mathbb{R}_{+}^{n}$, if $B_{i}(x)=B_{i}(y)$ and $x_{j}=y_{j}$ for all $j \in B_{i}(x) \cup\{i\}$, then

$$
D_{i}(x)=D_{i}(y)
$$

Normalization is a standard axiom in the literature. This rather mild condition requires that individual deprivation assumes a value of zero if and only if there are no individuals with a higher income than $i$. This property rules out the degenerate index that assigns the same deprivation value to all income distributions.

Normalization. For all $x \in \mathbb{R}_{+}^{n}$,

$$
D_{i}(x)=0 \Leftrightarrow B_{i}(x)=\emptyset .
$$

Translation invariance requires that the deprivation index is an absolute measure, that is, individual deprivation is invariant with respect to equal absolute changes in all incomes.

Translation invariance. For all $x \in \mathbb{R}_{+}^{n}$ and for all $\delta \in \mathbb{R}$ such that $\left(x+\delta \mathbf{1}^{n}\right) \in \mathbb{R}_{+}^{n}$,

$$
D_{i}\left(x+\delta \mathbf{1}^{n}\right)=D_{i}(x) .
$$

According to linear homogeneity, multiplying an income distribution by a positive number implies that the value of individual deprivation is multiplied by the same number.

Linear homogeneity. For all $x \in \mathbb{R}_{+}^{n}$ and for all $\lambda \in \mathbb{R}_{++}$,

$$
D_{i}(\lambda x)=\lambda D_{i}(x)
$$

The following axiom is a separability property that is responsible for the additive structure of our measures. Consider a decomposition of the set of those who are richer than $i$ into any two subgroups (unrelated to the exogenously given partition $S$ ). Let $x$ be any income distribution, and define two hypothetical distributions such that, in each, the individuals in one of the two subgroups are assigned the same income as person $i$ (and, therefore, do not contribute to the deprivation experienced by $i$ ). The condition demands that the deprivation value for $x$ can be obtained in an additive fashion using these hypothetical distributions for the two subgroups. This axiom appears in Bossert and D'Ambrosio (2006); see Ebert and Moyes (2000) for a similar but subtly different requirement. 
Additive decomposability. For all $x \in \mathbb{R}_{+}^{n}$ such that $B_{i}(x) \neq \emptyset$ and for all $B^{1}, B^{2} \subseteq$ $B_{i}(x)$, if $B^{1} \cap B^{2}=\emptyset$ and $B^{1} \cup B^{2}=B_{i}(x)$, then

$$
D_{i}(x)=D_{i}\left(\left.x_{i} \mathbf{1}^{n}\right|_{B^{1}},\left.x\right|_{N \backslash B^{1}}\right)+D_{i}\left(\left.x_{i} \mathbf{1}^{n}\right|_{B^{2}},\left.x\right|_{N \backslash B^{2}}\right) .
$$

The only axiom that utilizes the group structure induced by the partition $S$ requires that the index treat all individuals within a group impartially, paying no attention to their identities. This is a fundamental equal-treatment property with strong intuitive appeal in our setting.

Within-group anonymity. (a) For all $x \in \mathbb{R}_{+}^{n}$ and for all permutations $\pi$ of $S^{k_{i}}$ such that $\pi(i)=i$,

$$
D_{i}\left(x_{\pi}^{k_{i}},\left(x^{\ell}\right)_{\ell \in\{1, \ldots, K\} \backslash\left\{k_{i}\right\}}\right)=D_{i}(x) ;
$$

(b) for all $x \in \mathbb{R}_{+}^{n}$, for all $k \in\{1, \ldots, K\} \backslash\left\{k_{i}\right\}$ and for all permutations $\pi$ of $S^{k}$,

$$
D_{i}\left(x_{\pi}^{k},\left(x^{\ell}\right)_{\ell \in\{1, \ldots, K\} \backslash\{k\}}\right)=D_{i}(x) .
$$

In the degenerate case in which each individual is in a group by himself or herself (that is, if $K=n$ ), the axiom of within-group anonymity is vacuously satisfied and, therefore, the individuals need not be treated impartially because they all belong to different groups. Analogously, if the only group that has two members is group $S^{k_{i}}$ and each of the other groups has only one member (that is, if $K=n-1$ and $n^{k}=1$ for all $k \in\{1, \ldots, K\} \backslash\left\{k_{i}\right\}$ ), the axiom is trivially satisfied. In other words, the axiom is redundant if there exists no $j^{*} \in N$ such that $\left|S^{k_{j^{*}}} \backslash\{i\}\right|>1$. If there is but a single group (the case in which $K=1$ ), within-group anonymity reduces to the standard anonymity axiom and all individuals are to be treated impartially. This latter special case corresponds to the traditional framework without an externally imposed group structure.

The above axioms characterize our class of individual deprivation measures. All the properties related to individual deprivation are well-established and quite uncontroversial, while within-group anonymity is a very natural axiom in our group-dependent setup.

Theorem. A group-dependent individual deprivation index $D_{i}$ satisfies focus, normalization, translation invariance, linear homogeneity, additive decomposability and withingroup anonymity if and only if there exists $w_{i} \in \mathbb{R}_{++}^{K}$ such that $D_{i}=D_{i}^{w_{i}}$.

Proof. That the $D_{i}^{w_{i}}$ satisfy the axioms of the theorem statement is straightforward to verify.

Conversely, suppose that $D_{i}$ satisfies the axioms. Consider first distributions of the form $\left(\left.x_{j} \mathbf{1}^{n}\right|_{\{j\}},\left.x_{i} \mathbf{1}^{n}\right|_{N \backslash\{j\}}\right)$ where $j \in N \backslash\{i\}$ and $x_{j}>x_{i}$. Translation invariance with $\delta=-x_{i}$ implies

$$
D_{i}\left(\left.x_{j} \mathbf{1}^{n}\right|_{\{j\}},\left.x_{i} \mathbf{1}^{n}\right|_{N \backslash\{j\}}\right)=D_{i}\left(\left.\left(x_{j}-x_{i}\right) \mathbf{1}^{n}\right|_{\{j\}},\left.\mathbf{0}^{n}\right|_{N \backslash\{j\}}\right) .
$$

Let $f_{i}^{j}\left(x_{j}-x_{i}\right)=D_{i}\left(\left.\left(x_{j}-x_{i}\right) \mathbf{1}^{n}\right|_{\{j\}},\left.\mathbf{0}_{n}\right|_{N \backslash\{j\}}\right)$. Linear homogeneity with $\lambda=1 /\left(x_{j}-x_{i}\right)$ implies $f_{i}^{j}\left(x_{j}-x_{i}\right)=f_{i}^{j}(1)\left(x_{j}-x_{i}\right)$. Substituting back and defining $\bar{w}_{i}^{j}=f_{i}^{j}(1)$, we obtain

$$
D_{i}\left(\left.x_{j} \mathbf{1}^{n}\right|_{\{j\}},\left.x_{i} \mathbf{1}^{n}\right|_{N \backslash\{j\}}\right)=\bar{w}_{i}^{j}\left(x_{j}-x_{i}\right) .
$$


Now let $x \in \mathbb{R}_{+}^{n}$ be arbitrary. If $B_{i}(x)=\emptyset, D_{i}(x)=0=D_{i}^{w_{i}}(x)$ for any choice of $w_{i} \in \mathbb{R}_{++}^{K}$ follows immediately from normalization. If $B_{i}(x) \neq \emptyset$, the focus axiom allows us to assume that, without loss of generality, $x_{j}=x_{i}$ for all $j \in N \backslash B_{i}(x)$. Focus and repeated application of additive decomposability together imply

$$
D_{i}(x)=\sum_{i \in B_{i}(x)} D_{i}\left(\left.x_{j} \mathbf{1}^{n}\right|_{\{j\}},\left.x_{i} \mathbf{1}^{n}\right|_{N \backslash\{j\}}\right)
$$

and, by (2), we obtain

$$
D_{i}(x)=\sum_{j \in B_{i}(x)} \bar{w}_{i}^{j}\left(x_{j}-x_{i}\right) .
$$

Let $k \in\{1, \ldots, K\}$ and $j, \ell \in S^{k}$. By within-group anonymity, $\bar{w}_{i}^{j}$ and $\bar{w}_{i}^{\ell}$ can be chosen to be equal. Define $w_{i}^{k}=\bar{w}_{i}^{j}$, where $j$ is any arbitrary member of $S^{k}$. Thus, (3) implies

$$
D_{i}(x)=\sum_{k=1}^{K} w_{i}^{k} \sum_{j \in B_{i}^{k}\left(x^{k}, x_{i}\right)}\left(x_{j}-x_{i}\right)=D_{i}^{w_{i}}(x) .
$$

The axioms used in the above characterization are independent, as established by means of the following examples. For each of them, the axiom that is not satisfied is indicated.

Focus. For all $x \in \mathbb{R}_{+}^{n}$,

$$
D_{i}(x)=\left(n-\left|W_{i}(x)\right|\right) \sum_{j \in B_{i}(x)}\left(x_{j}-x_{i}\right) .
$$

Normalization. For all $x \in \mathbb{R}_{+}^{n}$,

$$
D_{i}(x)=0 .
$$

Translation invariance. For all $x \in \mathbb{R}_{+}^{n}$,

$$
D_{i}(x)=\sum_{j \in B_{i}(x)} \sqrt{x_{j}^{2}-x_{i}^{2}}
$$

Linear homogeneity. For all $x \in \mathbb{R}_{+}^{n}$,

$$
D_{i}(x)=\sum_{j \in B_{i}(x)}\left(x_{j}-x_{i}\right)^{2} .
$$

Additive decomposability. For all $x \in \mathbb{R}_{+}^{n}$,

$$
D_{i}(x)=\sqrt{\sum_{j \in B_{i}(x)}\left(x_{j}-x_{i}\right)^{2}} .
$$


Within-group anonymity. Let $j^{*} \in N$ be such that $\left|S^{k^{*}} \backslash\{i\}\right|>1$ (recall that withingroup anonymity is redundant in all other cases). For all $x \in \mathbb{R}_{+}^{n}$,

$$
D_{i}(x)= \begin{cases}\sum_{j \in B_{i}(x) \backslash\left\{j^{*}\right\}}\left(x_{j}-x_{i}\right)+2\left(x_{j^{*}}-x_{i}\right) & \text { if } j^{*} \in B_{i}(x), \\ \sum_{j \in B_{i}(x)}\left(x_{j}-x_{i}\right) & \text { if } j^{*} \notin B_{i}(x) .\end{cases}
$$

It is common in the literature to define an aggregate measure of deprivation as a weighted sum of the individual indices. If comparisons across time periods or societies involving different population sizes have to be performed, a weighted arithmetic mean rather than a sum is employed. Because we want to treat individuals anonymously within each group, the vectors of weights we use are not permitted to vary across individuals within the same group. Therefore, for each $i \in N$, the weights that express $i$ 's link to the various groups depend on $k_{i}$ rather than $i$ and, therefore, we require merely $K$ vectors of weights $r_{1}, \ldots, r_{K} \in \mathbb{R}_{++}^{K}$ in place of the $n$ vectors $w_{1}, \ldots, w_{n} \in \mathbb{R}_{++}^{K}$ to capture the relevant information in the group-dependent environment considered here. This allows us to arrange the requisite parameter values in a $K \times K$ matrix $R=\left(r_{\ell}^{k}\right)_{k, \ell \in\{1, \ldots, K\}}$. Using this matrix, the class of aggregate group-dependent measures of deprivation $D^{R}: \mathbb{R}_{+}^{n} \rightarrow \mathbb{R}_{+}$ corresponding to the individual measures characterized earlier is given by the sum of the individual deprivation measures so that

$$
D^{R}(x)=\sum_{i=1}^{n} \sum_{k=1}^{K} \sum_{j \in B_{i}^{k}\left(x^{k}, x_{i}\right)} r_{k_{i}}^{k}\left(x_{j}-x_{i}\right)
$$

for all $x \in \mathbb{R}_{+}^{n}$. As is the case for the individual measures, the arithmetic mean is obtained by a suitable choice of the weights $r_{\ell}^{k}$.

An important class of index numbers covered by our analysis is obtained if everyone in the same group $S^{k}$ has the same income $y_{k}$-that is, if the distribution $x$ is given by $x=\left(y_{1} \mathbf{1}^{n^{1}}, \ldots, y_{K} \mathbf{1}^{n^{K}}\right)$. In this case, the domain of $D^{R}$ is restricted to the set of income distributions with that property; note that the partition $\left\{S^{1}, \ldots, S^{K}\right\}$ is exogenously given. On this limited domain, (4) can be written as

$$
D^{R}(y)=\sum_{l=1}^{K} \sum_{\substack{k \in\{1, \ldots, K\}: \\ y_{k}>y_{\ell}}} r_{\ell}^{k} n^{\ell} n^{k}\left(y_{k}-y_{\ell}\right)
$$

for all $y \in \mathbb{R}_{+}^{K}$.

\section{$3 \quad$ Special cases}

The general class of aggregate deprivation measures proposed in this paper encompasses numerous other social index numbers, proposed in frameworks other than deprivation, that are based on differences or, more generally, on distances. We begin with two measures that emerge quite naturally because, as is the case for measures of deprivation, they are 
based on a one-dimensional variable with the agreed-upon property that more of it is better than less.

The first prominent example consists of the class of income-polarization indices proposed by Esteban and Ray (1994). Groups play a key role in this literature and it is assumed that everyone in the same group has the same income; this means that the framework described in (5) applies. Polarization in a society is intended to measure the degree to which the population is clustered in different groups composed of identical individuals, and how far apart these groups are. According to the alienation-identification framework introduced by Esteban and Ray, each individual feels alienated from others and this sentiment of alienation is mediated by a weight capturing the identification among the members of the same group. This weight is given by the number of individuals in the group and is raised to a power $\alpha$ capturing the importance identification plays. As opposed to deprivation, alienation is symmetric and is a sentiment experienced with respect to both richer and poorer individuals. The interaction of alienation and identification gives rise to effective antagonism, and polarization is postulated to be the sum of all effective antagonisms. Hence, the resulting polarization measures can be expressed as

$$
\begin{aligned}
P(y) & =d \sum_{\ell=1}^{K} \sum_{k=1}^{K}\left(n^{\ell}\right)^{1+\alpha} n^{k}\left|y_{\ell}-y_{k}\right| \\
& =d \sum_{\ell=1}^{K}\left(\sum_{\substack{k \in\{1, \ldots, K\}: \\
y_{k}>y_{\ell}}}\left(n^{\ell}\right)^{1+\alpha} n^{k}\left(y_{k}-y_{\ell}\right)+\sum_{\substack{k \in\{1, \ldots, K\}: \\
y_{k}<y_{\ell}}}\left(n^{\ell}\right)^{1+\alpha} n^{k}\left(y_{\ell}-y_{k}\right)\right) \\
& =d \sum_{\ell=1}^{K} \sum_{\substack{k \in\{1, \ldots, K\}: \\
y_{k}>y_{\ell}}}\left[\left(n^{\ell}\right)^{\alpha}+\left(n^{k}\right)^{\alpha}\right] n^{\ell} n^{k}\left(y_{k}-y_{\ell}\right)
\end{aligned}
$$

for all $y \in \mathbb{R}_{+}^{K}$ with a positive constant $d$ and a parameter $\alpha \in\left(0, \alpha^{*}\right]$, where $\alpha^{*}$ is approximately equal to 1.6; see Esteban and Ray (1994, p. 833). Setting $r_{\ell}^{k}=d\left[\left(n^{\ell}\right)^{\alpha}+\left(n^{k}\right)^{\alpha}\right]$ for all $k, \ell \in\{1, \ldots, K\}$ in (5), it follows that (6) emerges as a special case of our aggregate index.

Esteban and Ray (1994) do not include the parameter value of $\alpha=0$ because they focus on polarization rather than the measurement of income inequality, where identification plays no role. Nevertheless, this parameter value is also accommodated by our class, and it leads to the well-known absolute Gini index of inequality, a measure of dispersion around the global mean of the distribution as opposed to polarization where the focus is on clusters on local means; see Gini (1912). Substituting $\alpha=0$ in (6), we obtain a multiple of the absolute Gini coefficient $I$ defined by

$$
I(y)=d \sum_{\ell=1}^{K} \sum_{k=1}^{K} n^{\ell} n^{k}\left|y_{\ell}-y_{k}\right|=2 d \sum_{\ell=1}^{K} \sum_{\substack{k \in\{1, \ldots, K\}: \\ y_{k}>y_{\ell}}} n^{\ell} n^{k}\left(y_{k}-y_{\ell}\right)
$$

for all $y \in \mathbb{R}_{+}^{K}$. Clearly, (7) results from setting $r_{\ell}^{k}=2 d$ for all $k, \ell \in\{1, \ldots, K\}$ in (5). 
We now move on to examples for which it is not immediately apparent that they can be generated from our general class, starting with indices of fractionalization that are intended to capture the degree to which a society is split into distinct groups. As a first instance, we observe that measures of fractionalization (see Mauro, 1995, among many others) are of a somewhat different structure than the indices discussed so far. In particular, they are typically defined in terms of the cardinalities of the constituent groups of a partition - or, equivalently, in terms of the population shares of the groups. Because we treat this partition and the population shares as exogenously given, we express these measures as the resulting numbers. There are no incomes explicitly involved in these definitions (they may, of course, appear implicitly if they determine membership in a group) but, instead, the distances between these groups are taken into consideration. Because of this crucial difference, the measures discussed in this section are not special cases of ours in the literal sense but, as will become clear shortly, they certainly are special cases in the spirit of our approach. To be more precise, in order to express these fractionalization measures as special cases of our class, we replace the income differences $\left|y_{\ell}-y_{k}\right|$ with the distances $\delta(\ell, k)$ between group $\ell$ and group $k$ for all $\ell, k \in\{1, \ldots, K\}$. We assume that the distance function $\delta:\{1, \ldots, K\}^{2} \rightarrow \mathbb{R}_{+}$is such that $\delta(\ell, \ell)=0$ for all $\ell \in\{1, \ldots, K\}$ and $\delta(\ell, k)=\delta(k, \ell)$ for all $\ell, k \in\{1, \ldots, K\}$. Thus, we require the distance between a group and itself to be zero and the notion of distance to be symmetric - the distance from one group to another is the same as the distance from the latter to the former. The distance function $\delta$ need not be a metric. This is the case because the triangle inequality is not an essential property in the relevant literature. Moreover, we allow for distance functions that assign a distance of zero to distinct groups because some approaches in the literature exhibit this feature and we want to accommodate them here.

The vast majority of fractionalization measures that appear in the literature are special cases of the following general expression

$$
F=a \sum_{\ell=1}^{K} \sum_{k=1}^{K}\left(n^{\ell}\right)^{\beta}\left(n^{k}\right)^{\gamma} \delta(\ell, k)
$$

where $a \in \mathbb{R}_{++}$and $\beta, \gamma \in \mathbb{R}_{+}$are parameters. In analogy to the indices of polarization and inequality discussed earlier, (8) can be rewritten as

$$
F=a \sum_{\ell=1}^{K} \sum_{\substack{k \in\{1, \ldots, K\}: \\ k>\ell}}\left[\left(n^{\ell}\right)^{\beta-1}\left(n^{k}\right)^{\gamma-1}+\left(n^{k}\right)^{\beta-1}\left(n^{\ell}\right)^{\gamma-1}\right] n^{\ell} n^{k} \delta(\ell, k) .
$$

It follows immediately that (9) is obtained from replacing $\left(y_{k}-y_{\ell}\right)$ with $\delta(\ell, k)$ and substituting $r_{\ell}^{k}=a\left[\left(n^{\ell}\right)^{\beta-1}\left(n^{k}\right)^{\gamma-1}+\left(n^{k}\right)^{\beta-1}\left(n^{\ell}\right)^{\gamma-1}\right]$ in (5).

The class in (9) encompasses numerous indices that appear in the literature. First, consider the Gini-Simpson index (Gini, 1912; Simpson, 1949), also known as Greenberg's (1956) A-index, defined by

$$
A=\frac{1}{n^{2}} \sum_{\ell=1}^{K} \sum_{\substack{k \in\{1, \ldots, K\}: \\ k \neq \ell}} n^{\ell} n^{k}=\frac{2}{n^{2}} \sum_{\ell=1}^{K} \sum_{\substack{k \in\{1, \ldots, K\}: \\ k>\ell}} n^{\ell} n^{k}
$$


which is obtained by setting $a=2 / n^{2}$ and $\beta=\gamma=1$ in (9). The distance function in this case is given by the discrete metric that assigns a value of one to all pairs of distinct groups $(\ell, k)$ and, therefore, distances between different groups are not taken into account. This index can be expressed as one minus the Herfindahl-Hirschman index. The Gini-Simpson index measures the probability that two randomly chosen individuals in a society belong to different groups. In the framework of an ethno-linguistic partition of the population, this index is often referred to as ELF (ethno-linguistic fractionalization).

The index of Davydov and Weber (2016), defined as

$$
D W=\frac{1}{n^{2}} \sum_{\ell=1}^{K} \sum_{\substack{k \in\{1, \ldots, K\}: \\ k \neq \ell}}\left(n^{\ell}\right)^{\beta} n^{k}=\frac{1}{n^{2}} \sum_{\ell=1}^{K} \sum_{\substack{k \in\{1, \ldots, K\}: \\ k>\ell}}\left[\left(n^{\ell}\right)^{\beta-1}+\left(n^{k}\right)^{\beta-1}\right] n^{\ell} n^{k},
$$

is the special case of (9) that emerges if we use the parameter values $a=1 / n^{2}$ and $\gamma=1$, again employing the discrete metric. The role played by the parameter $\beta$ is analogous to that of $\alpha$ in the context of polarization to reflect the strength of individuals' identification with members of their own group. Note that the Davydov-Weber index is equivalent to the biodiversity measure proposed by Hill (1973).

The index of Reynal-Querol (2002) is an index of social polarization derived within Esteban and Ray's (1994) framework. It intends to capture how far the distribution of the different groups is from the bipolar distribution, and it is obtained for the parameter values $a=1 / n^{2}, \beta=2$ and $\gamma=1$. Again, distances between groups are not taken into account. This is not ideal since some groups may be more similar among themselves than others and one may want to incorporate this observation in the measurement of fractionalization and social polarization. For example, if the population is composed of linguistic groups, it makes perfect sense to say that Italian and Spanish are more similar to each other than either of them is to German. In this case, the use of a linguistic distance will allow to differentiate among pairs of distinct groups without automatically assigning a value of one.

The B-index of Greenberg (1956), defined as

$$
B=\frac{1}{n^{2}} \sum_{\ell=1}^{K} \sum_{k=1}^{K} n^{\ell} n^{k} \delta(\ell, k)=\frac{2}{n^{2}} \sum_{\ell=1}^{K} \sum_{\substack{k \in\{1, \ldots, K\}: \\ k>\ell}} n^{\ell} n^{k} \delta(\ell, k),
$$

goes in this direction and generalizes the A-index by allowing for a more general distance function. This index is, again, obtained by setting $a=2 / n^{2}$ and $\beta=\gamma=1$ in (9). In contrast to the $\mathrm{A}$-index, the distance function $\delta$ need not be the discrete metric. The index $B$ is also known as the quadratic-entropy index discussed by Rao (1982) and Rao and Nayak (1985). Unlike Greenberg (1956), the latter two contributions provide axiomatizations of this measure. See also Bossert, D'Ambrosio and La Ferrara (2011) for a generalized index of ethno-linguistic fractionalization labeled GELF.

Likewise, the generalization of the Davydov-Weber index is given by

$$
G D W=\frac{1}{n^{2}} \sum_{\ell=1}^{K} \sum_{k=1}^{K}\left(n^{\ell}\right)^{\beta} n^{k} \delta(\ell, k)
$$




$$
=\frac{1}{n^{2}} \sum_{\ell=1}^{K} \sum_{\substack{k \in\{1, \ldots, K\}: \\ k>\ell}}\left[\left(n^{\ell}\right)^{\beta-1}+\left(n^{k}\right)^{\beta-1}\right] n^{\ell} n^{k} \delta(\ell, k)
$$

and it again results from using the parameter values $a=1 / n^{2}$ and $\gamma=1$ with a general distance function in (9).

A variation of Greenberg's B-index is proposed by Desmet, Ortuño-Ortín and Weber (2017) to measure peripheral heterogeneity. It applies to situations in which there is a dominant group and it takes into account the alienation between the center and the peripheral groups, but not between the peripheral groups themselves. As the authors note, the index of peripheral heterogeneity can be viewed as an intermediate index between fractionalization and polarization. This is an instance of an index that requires the value of $\delta$ to be equal to zero for some pairs of distinct groups. Assuming, without loss if generality, that group 1 is the dominant group and the distances between any two groups that are different from the dominant group (referred to as peripheral groups) are zero, the center-periphery index is given by

$$
C P=\frac{1}{n^{2}} \sum_{k=2}^{K} n^{1} n^{k} \delta(1, k)
$$

which, with a distance function $\delta$ such that $\delta(\ell, k)=0$ for all $\ell, k \in\{1, \ldots, K\}$ such that $\ell \neq 1$ and $k \neq 1$, can equivalently be written as

$$
C P=\frac{1}{n^{2}} \sum_{\ell=1}^{K} \sum_{\substack{k \in\{1, \ldots, K\}: \\ k>\ell}} n^{\ell} n^{k} \delta(\ell, k) .
$$

It follows that $C P$ is obtained by substituting $a=2 / n^{2}$ and $\beta=\gamma=1$ in (9) and, in addition, employing a distance function that assigns a positive distance only if one of the two groups in the pair $(\ell, k)$ is given by the dominant group - that is, group 1. Of course, a special case is obtained if $\delta$ is chosen to be the discrete metric.

The following two groups of measures are hybrid in the sense that they combine information on social diversity (such as ethnicity) with income differences; for this reason, they are labeled indices of diversity-inequality and of diversity-polarization. The former is a generalization of the absolute Gini index of inequality, the latter a generalization of the Esteban-Ray polarization measure.

Suppose there is a finite number $Q \geq 2$ of ethnic groups $\{1, \ldots, Q\}$ and the pairwise distance between two of these groups $q$ and $q^{\prime}$ is expressed by means of a distance function $\phi:\{1, \ldots, Q\}^{2} \rightarrow \mathbb{R}_{+}$with the properties that $\phi\left(q, q^{\prime}\right)=0$ if and only if $q=q^{\prime}$ and $\phi\left(q, q^{\prime}\right)=\phi\left(q^{\prime}, q\right)$ for all $q, q^{\prime} \in\{1, \ldots, Q\}$. That is, any two distinct groups have a positive distance and the distance function is symmetric. Suppose, furthermore, that there are $M \geq 2$ distinct income levels $y_{1}, \ldots, y_{M}$ such that, without loss of generality, $y_{1}<\ldots<y_{M}$. The partition $S=\left\{S^{1}, \ldots, S^{K}\right\}$ is composed of $K=M Q$ groups. The members of group $S^{k}$ have income level $y_{m}$ and belong to the ethnic group $q$. Again without loss of generality, suppose that $S^{1}$ corresponds to $(1,1), S^{2}$ corresponds to $(1,2)$ 
etc. so that $S^{K}$ corresponds to $(M, Q)$. This convention allows us to identify, for any $k \in\{1, \ldots, K\}$, the unique generating pair $(\bar{m}(k), \bar{q}(k))$ that defines membership in the $k^{\text {th }}$ group. To complete the description of the basic setup, we define the distance that applies to the groups $S^{1}, \ldots, S^{K}$ by letting $\delta(\ell, k)=\phi(\bar{q}(\ell), \bar{q}(k))$ for all $\ell, k \in\{1, \ldots, K\}$.

The diversity-inequality index (see Desmet, Le Breton, Ortuño-Ortín and Weber, 2011, Chakravarty, 2015, and Hodler, Srisuma, Vesperoni and Zurlinden, 2020) is defined by

$$
\begin{aligned}
D I(y) & =d \sum_{\ell=1}^{K} \sum_{k=1}^{K} n^{\ell} n^{k}\left|y_{\ell}-y_{k}\right|[1+\delta(\ell, k)] \\
& =2 d \sum_{\ell=1}^{K} \sum_{\substack{k \in\{1, \ldots, K\}: \\
y_{k}>y_{\ell}}} n^{\ell} n^{k}\left(y_{k}-y_{\ell}\right)[1+\delta(\ell, k)]
\end{aligned}
$$

for all $y \in \mathbb{R}_{+}^{K}$, where $d$ is a positive constant. Thus, we can obtain $D I$ as a special case of (5) by setting the requisite parameter values $r_{\ell}^{k}$ equal to $d[1+\delta(\ell, k)]$. The index reduces to the Gini if $\delta(\ell, k)=0$ and, therefore, $D I$ represents an extension of (7) to a setting where diversity is taken into consideration.

We conclude this section with a proposal of a natural extension of the Esteban and Ray (1994) index of polarization that obtains if we choose the parameters $r_{\ell}^{k}=$ $d\left[\left(n^{\ell}\right)^{\alpha}+\left(n^{k}\right)^{\alpha}\right][1+\delta(\ell, k)]$ for all $k, \ell \in\{1, \ldots, K\}$ in $(5)$. Replacing the original values in (6) accordingly, we can define a diversity-polarization index as

$$
D P(y)=d \sum_{\ell=1}^{K} \sum_{\substack{k \in\{1, \ldots, K\}: \\ y_{k}>y_{\ell}}}\left[\left(n^{\ell}\right)^{\alpha}+\left(n^{k}\right)^{\alpha}\right] n^{\ell} n^{k}\left(y_{k}-y_{\ell}\right)[1+\delta(\ell, k)]
$$

for all $y \in \mathbb{R}_{+}^{K}$ so that Esteban and Ray's (1994) index results if $\delta(\ell, k)=0$.

The above two indices rely on the assumption that individuals in the same group have the same level of income. Along the lines discussed in Duclos, Esteban and Ray (2004, p. 1760), several additional generalizations are possible. If we allow for differences within groups, a more general distance measure between the requisite within-group distributions can be employed; this may move us beyond our current framework that is based on pairwise distances. An example of such a measure is the Kolmogorov index of variation distance, which is employed for the measures of social distance proposed by D'Ambrosio (2001).

\section{Concluding remarks}

We focus on absolute measures in this paper because of their prominence in the field of deprivation measurement. As is standard in the literature, our observations can easily be extended to relative measures by taking logarithms.

The characterization result of Section 2 can be generalized. If linear homogeneity is removed from the list of axioms, the remaining properties characterize the class of individual group-dependent deprivation measures that are obtained by replacing the constants 
$w_{i}^{k}$ in (1) with functions $f_{i}^{k}$ of the differences $x_{j}-x_{i}$. If translation invariance is dropped in addition to linear homogeneity, the $f_{i}^{k}$ are, in turn, replaced with functions $g_{i}^{k}$ of the pairs $\left(x_{j}, x_{i}\right)$. We do not state the axiomatizations of these more general classes explicitly because all of the indices discussed here are special cases of the measures that we do characterize. Details are available from the authors on request.

There also is a link to the measurement of communication benefits in a multi-lingual community, although it is less immediate than is the case for fractionalization indices. To illustrate, suppose there are a finite number $L \geq 2$ of languages, and a linguistic profile consists of a non-empty subset of these languages (see, for instance, Ginsburgh and Weber, 2020, for a comprehensive treatment). Thus, the set of all possible profiles has $K=2^{L}-1$ elements $\left\{S^{1}, \ldots, S^{K}\right\}$. In contrast to our setting, however, these sets do not form a partition; clearly, the intersection of any two of these sets need not be empty. Letting $n^{k}$ denote the number of individuals with linguistic profile $S^{k}$ for all $k \in\{1, \ldots, K\}$, an aggregate measure of communication benefits is given by

$$
C B=\sum_{\ell=1}^{K} \sum_{k=1}^{K} n^{\ell} n^{k} r_{\ell}^{k}
$$

where

$$
r_{\ell}^{k}= \begin{cases}1 & \text { if } S^{\ell} \cap S^{k} \neq \emptyset \\ 0 & \text { if } S^{\ell} \cap S^{k}=\emptyset\end{cases}
$$

see, for example, Selten and Pool (1991), Church and King (1993), Lazear (1999) and Gabszewicz, Ginsburgh and Weber (2011). We note that (10) cannot be expressed as a special case of (9) - and, thus, of (5)-because, whenever $\ell=k$, the product $n^{k} n^{\ell}=\left(n^{k}\right)^{2}$ receives a weight of one as opposed to a weight of zero that applies in the framework considered elsewhere in this paper. Note that (10) can be rewritten as

$$
C B=2 \sum_{\ell=1}^{K} \sum_{\substack{k \in\{1, \ldots, K\}: \\ k>\ell}} n^{\ell} n^{k} r_{\ell}^{k}+\sum_{k=1}^{K}\left(n^{k}\right)^{2}
$$

which does not follow the pattern established earlier in the paper. Although a possible resolution of this discrepancy is, in principle, available by amending our basic setup so as to allow $k$ to be greater than or equal to $\ell$ in the scope of the second sum, this fix would still not address the non-partition issue that is inherent in the notion of communication benefits. Moreover, an approach of that nature would fail to allow for other proposals that are more elaborate than the index $C B$. For example, a common alternative to the choice of zero as a weight that applies to a pair of linguistic profiles with an empty intersection is to assign the minimal distance between the languages in the requisite profiles according to some distance measure defined on the languages themselves. Such a move would take us out of our framework because the role of the underlying languages could no longer be reduced to the set of linguistic profiles that they generate and, therefore, it would seem to constitute quite stretch to think of the resulting model as a special case of our approach - the $S^{k}$ could no longer be treated as primitives. 
An open question that remains is how our class can be further generalized to include indices where the weights are ordinal variables such as the health inequality measures proposed by Allison and Foster (2004) and the polarization indices for ordinal data introduced by Apouey (2007) and Permanyer and D'Ambrosio (2015).

\section{References}

Alesina, A., S. Michalopoulos and E. Papaioannou (2016), Ethnic inequality, Journal of Political Economy, 124, 428-488.

Allison, R. and J. Foster (2004), Measuring health inequality using qualitative data, Journal of Health Economics, 23, 505-524.

Apouey, B. (2007), Measuring health polarization with self-assessed health data, Health Economics, 16, 875-894.

Bossert, W. and C. D'Ambrosio (2006), Reference groups and individual deprivation, Economics Letters, 90, 421-426.

Bossert, W., C. D'Ambrosio and E. La Ferrara (2011), A generalized index of fractionalization, Economica, 78, 723-750.

Chakravarty, S. (2015), Inequality, Polarization and Conflict: An Analytical Study, New Delhi: Springer.

Church, J. and I. King (1993), Bilingualism and network externalities, Canadian Journal of Economics, 26, 337-345.

Clark, A.E. and C. D'Ambrosio (2015), Attitudes to income inequality: experimental and survey evidence, in A.B. Atkinson and F. Bourguignon, eds., Handbook of Income Distribution, Volume 2A, Amsterdam: North-Holland, pp. 1147-1208.

D'Ambrosio, C. (2001), Household characteristics and the distribution of income in Italy: an application of social distance measures, Review of Income and Wealth, 47, 43-64.

Davydov, D. and S. Weber (2016), A simple characterization of the family of diversity indices, Economics Letters, 147, 121-123.

Desmet, K., M. Le Breton, I. Ortuño-Ortín and S. Weber (2011), The stability and breakup of nations: a quantitative analysis, Journal of Economic Growth, 16, 183-213.

Desmet, K., I. Ortuño-Ortín and S. Weber (2017), Peripheral diversity: transfers versus public goods, Social Choice and Welfare, 49, 787-823.

Duclos, J.-Y., J. Esteban and D. Ray (2004), Polarization: concepts, measurement, estimation, Econometrica, 72, 1737-1772.

Ebert, U. and P. Moyes (2000), An axiomatic characterization of Yitzhaki's index of individual deprivation, Economics Letters, 68, 263-270.

Esteban, J.-M. and D. Ray (1994), On the measurement of polarization, Econometrica, $62,819-851$. 
Esteban, J. and D. Ray (2011), Linking conflict to inequality and polarization, American Economic Review, 101, 1345-1374.

Gabszewicz, J., V. Ginsburgh and S. Weber (2011), Bilingualism and communicative benefits, Annals of Economics and Statistics, 101/102, 271-286.

Gini, C. (1912), Variabilità e mutabilità, Contributo allo Studio delle Distribuzioni e delle Relazioni Statistiche, Bologna: C. Cuppini.

Ginsburgh, V. and S. Weber (2020), The economics of language, Journal of Economic Literature, 58, 348-404.

Greenberg, J. (1956), The measurement of linguistic diversity, Language, 32, 109-115.

Gurr, T.R. (1968), Psychological factors in civil violence, World Politics, 20, 245-278.

Herfindahl, O.C. (1950), Concentration in the Steel Industry. Unpublished PhD dissertation, Columbia University.

Hey, J.D. and P.J. Lambert (1980), Relative deprivation and the Gini coefficient: comment, Quarterly Journal of Economics, 95, 567-573.

Hill, M. (1973), Diversity and evenness: a unifying notation and its consequences, Ecology, 54, 427-432.

Hirschman, A.O. (1945), National Power and Structure of Foreign Trade. Berkeley: University of California Press.

Hodler, R., S. Srisuma, A. Vesperoni and N. Zurlinden (2020), Measuring ethnic stratification and its effect on trust in Africa, Journal of Development Economics, 146, forthcoming.

Kawachi, I., B.P. Kennedy and R.G. Wilkinson (1999), Crime: social disorganization and relative deprivation, Social Science \& Medicine, 48, 719-731.

Lazear, E. (1999), Culture and language, Journal of Political Economy, 107, S95-S126.

Mauro, P. (1995), Corruption and growth, Quarterly Journal of Economics, 110, 681-712.

Permanyer, I. and C. D'Ambrosio (2015), Measuring social polarization with ordinal and categorical data, Journal of Public Economic Theory, 17, 311-327.

Rao, C.R. (1982), Diversity and dissimilarity coefficients: a unified approach, Theoretical Population Biology, 21, 24-43.

Rao, C.R. and T.K. Nayak (1985), Cross entropy, dissimilarity measures and characterization of quadratic entropy, IEEE Transactions on Information Theory, 31, 589-593.

Reynal-Querol, M. (2002), Ethnicity, political systems, and civil wars, Journal of Conflict Resolution, 46, 29-54.

Runciman, W. (1966), Relative Deprivation and Social Justice, London: Routledge.

Selten, R. and J. Pool (1991), The distribution of foreign language skills as a game equilibrium, in R. Selten, ed., Game Equilibrium Models IV: Social and Political Interaction, Berlin: Springer, pp. 64-87. 
Sen, A. (1976), Poverty: an ordinal approach to measurement, Econometrica, 44, 219-231. Simpson, E.H. (1949), Measurement of diversity, Nature, 163, 688.

Stark, O. and J. Taylor (1989). Relative deprivation and international migration, Demography, 26, 1-14.

Yitzhaki, S. (1979), Relative deprivation and the Gini coefficient, Quarterly Journal of Economics, 93, 321-324.

Yitzhaki, S. (1980), Relative deprivation and the Gini coefficient: reply, Quarterly Journal of Economics, 95, 575-576. 